\title{
Computational study of scattering from healthy and diseased red blood cells
}

\author{
Özgür Ergül \\ University of Strathclyde \\ Department of Mathematics and Statistics \\ Glasgow, Scotland, G1 1XH \\ United Kingdom
}

\author{
Ayça Arslan-Ergül \\ Strathclyde Institute of Pharmacy and Biomedical Sciences \\ University of Strathclyde \\ Glasgow, Scotland, G4 ONR \\ United Kingdom
}

\section{Levent Gürel}

Bilkent University

Department of Electrical and Electronics Engineering and

Computational Electromagnetics Research Center (BiLCEM) TR-06800 Bilkent, Ankara

Turkey

\begin{abstract}
We present a comparative study of scattering from healthy red blood cells (RBCs) and diseased RBCs with deformed shapes. Scattering problems involving three-dimensional RBCs are formulated accurately with the electric and magnetic current combined-field integral equation and solved efficiently by the multilevel fast multipole algorithm. We compare scattering cross section values obtained for different RBC shapes and different orientations. In this way, we determine strict guidelines to distinguish deformed RBCs from healthy RBCs and to diagnose various diseases using scattering cross section values. The results may be useful for designing new and improved flow cytometry procedures. @ 2010 Society of Photo-Optical Instrumentation Engineers. [DOl: 10.1117/1.3467493]
\end{abstract}

Keywords: red blood cell; electromagnetic scattering; surface formulations; multilevel fast multipole algorithm.

Paper 09435R received Sep. 28, 2009; revised manuscript received Apr. 24, 2010; accepted for publication May 26, 2010; published online Aug. 5, 2010.

\section{Introduction}

Scattering from red blood cells (RBCs) has attracted the interest of many researchers in the area of biomedical science and engineering. Scattering cross section (SCS) values of an RBC provide essential information on the morphological properties of the cell. Hence, SCS values can be used to diagnose various diseases involving the deformation of RBCs. Using a diagnosis setup, i.e., a flow cytometer, a liquid stream including many RBCs from a blood sample can be passed through a thin tube, and SCS values of each RBC can be measured via an optical detection system. Then, measurement values can be compared with reference values to obtain statistical data on the size and the shape of RBCs in the blood sample under investigation. The goal of this study is to determine novel means of distinguishing diseased cells based on scattering statistics. Parameters of scattering scenarios are not limited to those of conventional cytometry setups.

Scattering problems involving a single RBC or multiple $\mathrm{RBCs}$ have been investigated extensively by employing various numerical methods, such as Mie-series approximations, ${ }^{1-5}$ Rayleigh-Gans and anomalous diffraction approximations, ${ }^{6-11}$ T-matrix approaches, ${ }^{12}$ finite-difference time-domain (FDTD) methods, ${ }^{13-15}$ and surface integral equations. ${ }^{16-20}$ In those studies, electromagnetic scattering or transmission characteristics of RBCs are investigated for different material properties, illumination angles, and frequencies. In addition, depending on the solution method, RBCs are assumed to be spherical, ellipsoidal, or biconcave, and their electromagnetic properties are considered as homogeneous or heterogeneous. Previous studies mostly focused on the simulation of healthy

Address all correspondence to: Özgür Ergül, University of Strathclyde, Department of Mathematics and Statistics, Glasgow, Scotland, G1 1XH, United Kingdom; Tel: 44141548 3650; E-mail: ozgur.ergul@strath.ac.uk
RBCs with ordinary shapes, and less attention has been paid to diseased RBCs with deformed shapes.

In this paper, we present a comparative study of scattering from healthy and deformed RBCs using surface integral equations. As depicted in Fig. 1, we consider a healthy (ordinary) $\mathrm{RBC}$ with a biconcave shape in addition to four types of deformed cells, namely, a spherocyte, a microcyte, a macrocyte, and a sickle cell (see Sec. 2 for detailed characteristics of these four deformed RBCs). For the five different RBC shapes, SCS values are calculated and compared. In this way, we determine strict guidelines to distinguish deformed RBCs from healthy RBCs and to diagnose various diseases using SCS values. Since SCS depends on the orientation of the $\mathrm{RBC}$, we also test all possible illuminations to avoid misinterpretations during the detection process.

Our simulation environment is based on the multilevel fast multipole algorithm ${ }^{21}$ (MLFMA), which provides efficient solutions of electromagnetics problems discretized with large numbers of unknowns. Problems are formulated with the electric and magnetic current combined-field integral equation ${ }^{22}$ (JMCFIE) discretized with the Rao-Wilton-Glisson ${ }^{23}$ (RWG) functions on planar triangles. JMCFIE provides wellconditioned matrix equations, which can be solved efficiently by using iterative algorithms. ${ }^{24}$ Nevertheless, accurate discretizations of $\mathrm{RBCs}$ at realistic frequencies lead to matrix equations involving hundreds of thousands of unknowns. Hence, we employ MLFMA to accelerate the matrix-vector multiplications (MVMs) required by iterative solvers without deteriorating the accuracy of results.

The paper is organized as follows. Section 2 provides an overview of possible deformations of RBCs due to various diseases. Section 3 presents rigorous formulations of scattering problems involving $\mathrm{RBCs}$, and their discretizations for

1083-3668/2010/15(4)/045004/8/\$25.00 (C) 2010 SPIE 


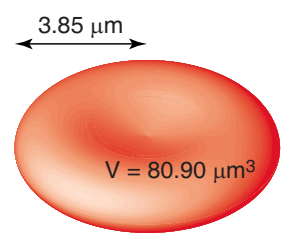

(a)

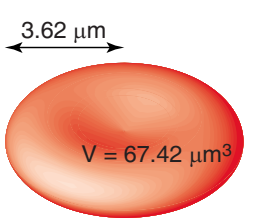

(c)

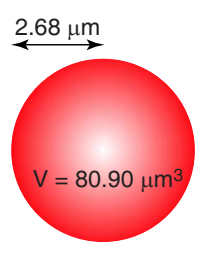

(b)

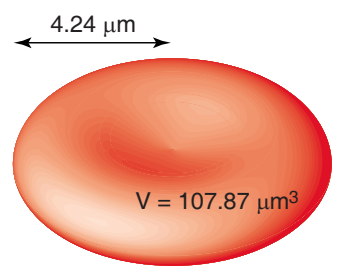

(d)

Fig. 1 Healthy and deformed RBCs: (a) a healthy (ordinary) RBC with a biconcave shape; (b) a spherocyte having exactly the same volume as the ordinary RBC; (c) a microcyte, which is smaller than an ordinary RBC, with a biconcave shape; (d) a macrocyte, which is larger than an ordinary RBC, with a biconcave shape; and (e) a sickle cell having exactly the same volume as an ordinary RBC.

numerical solutions. Efficient solutions of problems using iterative solvers, MLFMA, and preconditioners are presented in Sec. 4. Finally, Sec. 5 presents solutions and numerical results, followed by our concluding remarks in Sec. 6.

\section{Deformation of Red Blood Cells}

Due to various diseases, RBCs can be deformed in terms of size and shape. For example, an RBC is identified ${ }^{25}$ as a macrocyte if its diameter is larger than $8.5 \mu \mathrm{m}$, even though it may have a biconcave shape like an ordinary RBC. Similarly, an RBC with a diameter smaller than $7.0 \mu \mathrm{m}$ is identified as a microcyte. ${ }^{25}$ Among various types of shape-deformed RBCs, sickle cells are identified by their extraordinary thin and long structures with pointed ends, ${ }^{26}$ whereas spherocytes have almost spherical geometries. ${ }^{27}$

Microcytosis and macrocytosis have clinical significance because they may indicate other serious underlying diseases. Microcytosis is observed mainly in disorders in iron metabolism or deficiencies in hemoglobin synthesis, ${ }^{28}$ whereas macrocytosis is mostly observed in drug use, alcoholism, liver diseases, myeloma, and leukemia. ${ }^{29}$ On the other hand, sickle cells are encountered in sickle-cell anemia or sickle-cell trait, which are caused by abnormal hemoglobin structures. Due to their extraordinary shape, sickle cells may not be able to pass through capillaries, blocking the blood flow. ${ }^{30,31}$ In the case of the sickle-cell anemia, 30 to $60 \%$ of RBCs may have sickle shapes, whereas this ratio drops to only $1 \%$ for the sickle-cell trait. ${ }^{26}$ In general, the number of sickle cells depends on various factors, particularly deoxygenation levels. ${ }^{26,30}$ Finally, spherocytes are commonly observed in hereditary spherocytosis disease, which further leads to anemia, splenomegaly, jaundice, and many other clinical symptoms. ${ }^{32}$

Currently, most automated diagnosis setups rely on mean corpuscular volume (MCV) to detect the deformation of RBCs in terms of size. Specifically, high and low MCV values may indicate the presence of macrocytes and microcytes, respectively. However, MCV measurements are usually insuffi- cient for a reliable diagnosis, and peripheral blood smears are required, especially in the early stages of macrocytosis. ${ }^{29}$ As opposed to microcytes and macrocytes, sickle cells can be diagnosed via biochemical and genetic tests, which are available only in specialized laboratories, ${ }^{25,33}$ and spherocytes are usually detected via mean corpuscular hemoglobin concentration and RBC width measurements. ${ }^{32}$ Nevertheless, blood smear tests remain as the gold standard of diagnosing sickle cells and spherocytes, as for microcytes and macrocytes.

As presented in this paper, ordinary and deformed RBCs, i.e., microcytes, macrocytes, sickle cells, and spherocytes, can be distinguished from each other using a complete analysis of SCS values. Based on our results, we offer a fast and reliable technique to detect deformed RBCs in a blood sample using an automated diagnosis setup.

\section{Formulation}

Consider a homogeneous dielectric object with a threedimensional arbitrary shape located in a homogeneous space. Electromagnetic permittivity and permeability of the object and the outer medium are $\left(\epsilon_{i}, \mu_{i}\right)$ and $\left(\epsilon_{o}, \mu_{o}\right)$, respectively. Applying the equivalence principle, ${ }^{34}$ equivalent electric and magnetic currents are defined on the surface of the object $S$, i.e.,

$$
\begin{gathered}
\mathbf{J}(\mathbf{r})=\hat{n} \times \mathbf{H}(\mathbf{r}), \\
\mathbf{M}(\mathbf{r})=-\hat{n} \times \mathbf{E}(\mathbf{r}),
\end{gathered}
$$

where $\hat{n}$ is the unit normal vector. Using the equivalent currents, secondary (scattered) electric and magnetic fields can be calculated as

$$
\begin{aligned}
\mathbf{E}_{o, i}^{s}(\mathbf{r})= & \pm \eta_{o, i} \mathcal{I}_{o, i}(\mathbf{J})(\mathbf{r}) \mp \mathcal{K}_{o, i}^{\mathrm{PV}}(\mathbf{M})(\mathbf{r}) \\
& +\left(\Omega_{i, o} / 4 \pi\right) \hat{n} \times \mathbf{M}(\mathbf{r}),
\end{aligned}
$$


Ergül, Arslan-Ergül, and Gürel: Computational study of scattering from healthy and diseased red blood cells

$$
\begin{aligned}
\mathbf{H}_{o, i}^{s}(\mathbf{r})= & \pm \frac{1}{\eta_{o, i}} \mathcal{T}_{o, i}(\mathbf{M}, \mathbf{r}) \pm \mathcal{K}_{o, i}^{\mathrm{PV}}(\mathbf{J}, \mathbf{r}) \\
& -\left(\Omega_{i, o} / 4 \pi\right) \hat{n} \times \mathbf{J}(\mathbf{r}),
\end{aligned}
$$

when the observation point $\mathbf{r}$ is outside $(o)$ or inside $(i)$ the object. In Eqs. (3) and (4), $\eta_{u}=\left(\mu_{u} / \epsilon_{u}\right)^{1 / 2}$ for $u=i, o$ represents the wave impedance, $0 \leqslant \Omega_{o} \leqslant 4 \pi$ is the external solid angle, and $\Omega_{i}=4 \pi-\Omega_{o}$ is the internal solid angle. Integrodifferential operators are derived as

$$
\begin{aligned}
\mathcal{T}_{u}(\mathbf{X}, \mathbf{r})= & i k_{u} \int_{S} \mathrm{~d} \mathbf{r}^{\prime} X\left(\mathbf{r}^{\prime}\right) g_{u}\left(\mathbf{r}, \mathbf{r}^{\prime}\right) \\
& +\frac{i}{k_{u}} \int_{S} \mathrm{~d} \mathbf{r}^{\prime} \nabla^{\prime} \mathbf{X}\left(\mathbf{r}^{\prime}\right) \nabla g_{u}\left(\mathbf{r}, \mathbf{r}^{\prime}\right), \\
\mathcal{K}_{u}^{\mathrm{PV}}(\mathbf{X}, \mathbf{r})= & \int_{\mathrm{PV}, S} \mathrm{~d} \mathbf{r}^{\prime} \mathbf{X}\left(\mathbf{r}^{\prime}\right) \times \nabla^{\prime} g_{u}\left(\mathbf{r}, \mathbf{r}^{\prime}\right),
\end{aligned}
$$

where PV indicates the principal value of the integral, $k_{u}$ $=\omega\left(\mu_{u} \epsilon_{u}\right)^{1 / 2}$ is the wave number, and $g_{u}\left(r, r^{\prime}\right)$ denotes the homogeneous-space Green's function defined as

$$
g_{u}\left(\mathbf{r}, \mathbf{r}^{\prime}\right)=\frac{\exp \left(i k_{u} \mathrm{R}\right)}{4 \pi \mathrm{R}} \quad\left(\mathrm{R}=\left|\mathbf{r}-\mathbf{r}^{\prime}\right|\right),
$$

in phasor notation with the $\exp (-i \omega t)$ convention.

\subsection{JMCFIE}

Boundary conditions on the surface of the object can be written as

$$
\left\{\begin{array}{c}
\hat{n} \times \\
-\hat{n} \times \hat{n} \times
\end{array}\right\}\left(\mathbf{E}^{\mathrm{inc}}+\mathbf{E}_{o}^{s}\right)(\mathbf{r})=\left\{\begin{array}{c}
\hat{n} \times \\
-\hat{n} \times \hat{n} \times
\end{array}\right\} \mathbf{E}_{i}^{s}(\mathbf{r}),
$$

and

$$
\left\{\begin{array}{c}
\hat{n} \times \\
-\hat{n} \times \hat{n} \times
\end{array}\right\}\left(\mathbf{H}^{\mathrm{inc}}+\mathbf{H}_{o}^{s}\right)(\mathbf{r})=\left\{\begin{array}{c}
\hat{n} \times \\
-\hat{n} \times \hat{n} \times
\end{array}\right\} \mathbf{H}_{i}^{s}(\mathbf{r}),
$$

where $\mathbf{E}^{\text {inc }}(\mathbf{r})$ and $\mathbf{H}^{\text {inc }}(\mathbf{r})$ are incident electric and magnetic fields produced by external sources. Using Eqs. (3) and (4), and combining boundary conditions appropriately, JMCFIE is derived as

$$
\left[\begin{array}{ll}
\mathcal{Z}_{11} & \mathcal{Z}_{12} \\
\mathcal{Z}_{21} & \mathcal{Z}_{22}
\end{array}\right] \cdot\left[\begin{array}{c}
\mathbf{J} \\
\mathbf{M}
\end{array}\right](\mathbf{r})=\left[\begin{array}{l}
\mathbf{f}_{1} \\
\mathbf{f}_{2}
\end{array}\right](\mathbf{r})
$$

where

$$
\begin{aligned}
\mathcal{Z}_{11}(\mathbf{X}, \mathbf{r})= & -X(\mathbf{r})+\hat{n} \times\left(\mathcal{K}_{o}^{\mathrm{PV}}-\mathcal{K}_{i}^{\mathrm{PV}}\right)(\mathbf{X}, \mathbf{r}) \\
& -\hat{n} \times \hat{n} \times\left(\mathcal{T}_{o}+\mathcal{T}_{i}\right)(\mathbf{X}, \mathbf{r}), \\
\mathcal{Z}_{12}(\mathbf{X}, \mathbf{r})= & \hat{n} \times \hat{n} \times\left(\eta_{o}^{-1} \mathcal{K}_{o}^{\mathrm{PV}}+\eta_{i}^{-1} \mathcal{K}_{i}^{\mathrm{PV}}\right)(\mathbf{X}, \mathbf{r}) \\
& -(4 \pi)^{-1}\left(\eta_{o}^{-1} \Omega_{o}-\eta_{i}^{-1} \Omega_{i}\right) \hat{n} \times \mathbf{X}(\mathbf{r}) \\
& +\hat{n} \times\left(\eta_{o}^{-1} \mathcal{T}_{o}-\eta_{i}^{-1} \mathcal{T}_{i}\right)(\mathbf{X}, \mathbf{r}),
\end{aligned}
$$

$$
\begin{aligned}
\mathcal{Z}_{21}(\mathbf{X}, \mathbf{r})= & -\hat{n} \times \hat{n} \times\left(\eta_{o} \mathcal{K}_{o}^{\mathrm{PV}}+\eta_{i} \mathcal{K}_{i}^{\mathrm{PV}}\right)(\mathbf{X}, \mathbf{r}) \\
& +(4 \pi)^{-1}\left(\eta_{o} \Omega_{o}-\eta_{i} \Omega_{i}\right) \hat{n} \times \mathbf{X}(\mathbf{r}) \\
& -\hat{n} \times\left(\eta_{o} \mathcal{T}_{o}-\eta_{i} \mathcal{T}_{i}\right)(\mathbf{X}, \mathbf{r}), \\
& \mathcal{Z}_{22}(\mathbf{X}, \mathbf{r})=\mathcal{Z}_{11}(\mathbf{X}, \mathbf{r}),
\end{aligned}
$$

and

$$
\begin{aligned}
\mathbf{f}_{1}(\mathbf{r}) & =\eta_{o}^{-1} \hat{n} \times \hat{n} \times \mathbf{E}^{\mathrm{inc}}(\mathbf{r})-\hat{n} \times \mathbf{H}^{\mathrm{inc}}(\mathbf{r}), \\
\mathbf{f}_{2}(\mathbf{r}) & =\eta_{o} \hat{n} \times \hat{n} \times \mathbf{H}^{\mathrm{inc}}(\mathbf{r})+\hat{n} \times \mathbf{E}^{\mathrm{inc}}(\mathbf{r}) .
\end{aligned}
$$

Among infinitely many surface formulations of dielectric objects, JMCFIE is a preferable formulation in terms of efficiency and accuracy. ${ }^{22,24}$ Specifically, solutions of JMCFIE usually require fewer iterations than solutions of other formulations, particularly when the number of unknowns is large. ${ }^{24}$ In addition, JMCFIE provides more accurate solutions than other efficient formulations, such as the Müller formulation. ${ }^{24}$

\subsection{Discretization}

For numerical solutions of JMCFIE, equivalent currents are expanded in a series of basis functions $\mathbf{b}_{n}(\mathbf{r})$, i.e.,

$$
[\mathbf{J}(\mathbf{r}), \mathbf{M}(\mathbf{r})]=\sum_{n=1}^{N}\left[\mathbf{a}_{\mathbf{J}}(n), \mathbf{a}_{\mathbf{M}}(n)\right] \mathbf{b}_{n}(\mathbf{r}) .
$$

By testing JMCFIE using a set of functions $\mathbf{t}_{m}(\mathbf{r})$, we construct $2 N \times 2 N$ dense matrix equations in the form of

$$
\left[\begin{array}{ll}
\bar{Z}_{11} & \bar{Z}_{12} \\
\bar{Z}_{21} & \bar{Z}_{22}
\end{array}\right] \cdot\left[\begin{array}{l}
\mathbf{a}_{J} \\
\mathbf{a}_{M}
\end{array}\right]=\left[\begin{array}{l}
\mathbf{v}_{1} \\
\mathbf{v}_{2}
\end{array}\right]
$$

where

$$
\begin{aligned}
\mathbf{v}_{1}[m] & =\int_{S_{m}} \operatorname{drt}_{m}(\mathbf{r}) \cdot \mathbf{f}_{1}(\mathbf{r}) \\
& =-\int_{S_{m}} \operatorname{drt}_{m}(\mathbf{r}) \cdot\left(\eta_{o}^{-1} \mathbf{E}^{\mathrm{inc}}+\hat{n} \times \mathbf{H}^{\mathrm{inc}}\right)(\mathbf{r}),
\end{aligned}
$$

and

$$
\begin{aligned}
\mathbf{v}_{2}[m] & =\int_{S_{m}} \mathrm{~d} \mathbf{r} t_{m}(\mathbf{r}) \cdot \mathbf{f}_{2}(\mathbf{r}) \\
& =\int_{S_{m}} \mathrm{~d} \mathbf{r} \mathbf{t}_{m}(\mathbf{r}) \cdot\left(\hat{n} \times \mathbf{E}^{\mathrm{inc}}-\eta_{o} \mathbf{H}^{\mathrm{inc}}\right)(\mathbf{r}),
\end{aligned}
$$

The matrix elements in Eq. (18) are derived as

$$
\begin{gathered}
\overline{\mathrm{Z}}_{11}=-\overline{\mathrm{I}}+\overline{\mathrm{K}}_{o}^{\mathrm{PV}, \times n}-\overline{\mathrm{K}}_{i}^{\mathrm{PV}, \times n}+\overline{\mathrm{T}}_{o}+\overline{\mathrm{T}}_{i} \\
\overline{\mathrm{Z}}_{12}=-\eta_{o}^{-1} \overline{\mathrm{K}}_{o}^{\mathrm{PV}}-\eta_{i}^{-1} \overline{\mathrm{K}}_{i}^{\mathrm{PV}}-0.5\left(\eta_{o}^{-1}-\eta_{i}^{-1}\right) \overline{\mathrm{I}}^{\times n}+\eta_{o}^{-1} \overline{\mathrm{T}}_{o}^{\times n} \\
-\eta_{i}^{-1} \overline{\mathrm{T}}_{i}^{\times n}
\end{gathered}
$$


Ergül, Arslan-Ergül, and Gürel: Computational study of scattering from healthy and diseased red blood cells

$$
\begin{gathered}
\overline{\mathrm{Z}}_{21}=-\eta_{o} \overline{\mathrm{K}}_{o}^{\mathrm{PV}}+\eta_{i} \overline{\mathrm{K}}_{i}^{\mathrm{PV}}+0.5\left(\eta_{o}-\eta_{i}\right) \overline{\mathrm{I}}^{\times n} \\
-\eta_{o} \overline{\mathrm{T}}_{o}^{\times n}+\eta_{i} \overline{\mathrm{T}}_{i}^{\times n} \\
\overline{\mathrm{Z}}_{22}=\overline{\mathrm{Z}}_{11},
\end{gathered}
$$

where

$$
\begin{aligned}
& \overline{\mathrm{T}}_{u}[m, n]=\int_{S_{m}} \operatorname{drt}_{m}(\mathbf{r}) \cdot \mathcal{T}_{u}\left(\mathbf{b}_{n}, r\right), \\
& \overline{\mathbf{T}}_{u}^{\times n}[m, n]=\int_{S_{m}} \operatorname{drt}_{m}(\mathbf{r}) \cdot \hat{n} \times \mathcal{T}_{u}\left(\mathbf{b}_{n}, r\right), \\
& \overline{\mathrm{K}}_{u}^{\mathrm{PV}}[m, n]=\int_{S_{m}} \mathrm{drt}_{m}(\mathbf{r}) \cdot \mathcal{K}_{u}^{\mathrm{PV}}\left(\mathbf{b}_{n}, r\right), \\
& \overline{\mathrm{K}}_{u}^{\mathrm{PV}, \times n}[m, n]=\int_{S_{m}} \mathrm{~d} \mathbf{r} \mathbf{t}_{m}(\mathbf{r}) \cdot \hat{n} \times \mathcal{K}_{u}^{\mathrm{PV}}\left(\mathbf{b}_{n}, r\right), \\
& \bar{I}[m, n]=\int_{S_{m}} \mathrm{drt}_{m}(\mathbf{r}) \cdot \mathbf{b}_{n}(\mathbf{r}), \\
& \overline{\mathrm{T}}^{\times n}[m, n]=\int_{S_{m}} \mathrm{drt}_{m}(\mathbf{r}) \cdot \hat{n} \times \mathbf{b}_{n}(\mathbf{r}) .
\end{aligned}
$$

In Eqs. (19), (20), and (25)-(30), $S_{m}$ represents the spatial support of the $m$ th testing function. Using a Galerkin scheme, i.e., using the same set of RWG functions as basis and testing functions, $\mathcal{T}, \mathcal{K}$, and identity operators are well tested in Eqs. (25), (28), and (29), respectively.

\section{Iterative Solutions via MLFMA}

Matrix equations obtained from JMCFIE can be solved iteratively by employing a Krylov subspace algorithm. Among various methods available in the literature, we prefer the biconjugate-gradient-stabilized (BiCGStab) algorithm, ${ }^{35}$ which is known to provide rapid solutions for second-kind integral equations, such as JMCFIE. In each iteration, BiCGStab requires two MVMs, i.e.,

$$
\left[\begin{array}{l}
\mathbf{y}_{1} \\
\mathbf{y}_{2}
\end{array}\right]=\left[\begin{array}{ll}
\bar{Z}_{11} & \bar{Z}_{12} \\
\bar{Z}_{21} & \bar{Z}_{22}
\end{array}\right] \cdot\left[\begin{array}{l}
\mathbf{x}_{1} \\
\mathbf{x}_{2}
\end{array}\right] .
$$

For efficient solutions of scattering problems involving RBCs, we employ MLFMA to perform MVMs in $\mathcal{O}(N \log N)$ time using $\mathcal{O}(N \log N)$ memory.

\subsection{MLFMA for Dielectric Objects}

Considering Eq. (31), we perform four MVMs with the four partitions of the $2 N \times 2 N$ matrix. MVMs are decomposed as

$$
\overline{\mathrm{Z}}_{a b} \cdot \mathbf{x}_{b}=\overline{\mathrm{Z}}_{a b}^{\mathrm{NF}} \cdot \mathbf{x}_{b}+\overline{\mathrm{Z}}_{a b}^{\mathrm{FF}} \cdot \mathbf{x}_{b},
$$

for $a=1,2$ and $b=1,2$, where near-field interactions denoted by $\bar{Z}_{a b}^{\mathrm{NF}}$ are calculated directly and stored in memory to perform partial multiplications $\bar{Z}_{a b}^{\mathrm{NF}} \cdot \mathbf{x}_{b}$. On the other hand, multiplications involving far-field interactions, i.e., $\bar{Z}_{a b}^{\mathrm{FF}} \cdot \mathbf{x}_{b}$, are performed efficiently using MLFMA. Those multiplications are further decomposed into two parts as

$$
\overline{\mathrm{Z}}_{a b}^{\mathrm{FF}} \cdot \mathbf{x}_{b}=\overline{\mathrm{Z}}_{a b, o}^{\mathrm{FF}} \cdot \mathbf{x}_{b}+\overline{\mathrm{Z}}_{a b, i}^{\mathrm{FF}} \cdot \mathbf{x}_{b},
$$

since MLFMA is applied separately for inner and outer media.

To calculate far-field interactions, a multilevel tree structure with $L=\mathcal{O}(\log N)$ levels is constructed by placing the object in a cubic box and recursively dividing the computational domain into subdomains (clusters). In this way, interactions of basis and testing functions that are far from each other can be calculated efficiently using the factorization and the diagonalization of the Green's function. Each MVM in the form of $\bar{Z}_{a b, u}^{\mathrm{FF}} \cdot \mathbf{x}_{b}$ can be performed in three stages, called aggregation, translation, and disaggregation.

\subsubsection{Aggregation}

In this stage, radiated fields of clusters are calculated from the bottom of the tree structure to the highest level $(l=L)$. At the lowest level, radiation patterns of basis functions are multiplied with coefficients provided by the iterative solver and combined to obtain radiated fields of clusters, i.e.,

$$
\left\{\begin{array}{l}
\mathbf{S}_{a b, u}^{C, \theta} \\
\mathbf{S}_{a b, u}^{C, \phi}
\end{array}\right\}=\sum_{n \in C} \mathbf{x}_{b}[n]\left\{\begin{array}{l}
\mathbf{S}_{a b, u}^{n, \theta} \\
\mathbf{S}_{a b, u}^{n, \phi}
\end{array}\right\},
$$

where $\mathbf{S}_{a b, u}^{C, \theta}$ and $\mathbf{S}_{a b, u}^{C, \phi}$ are arrays of $K_{u}^{1}$ elements containing $\theta$ and $\phi$ components of the radiated field of a cluster $C$. Similarly, $\mathbf{S}_{a b, u}^{n, \theta}$ and $\mathbf{S}_{a b, u}^{n, \phi}$ in Eq. (34) are arrays of $K_{u}^{1}$ elements containing $\theta$ and $\phi$ components of the radiation pattern of the $n$th basis function. We note that radiation patterns of basis functions (hence radiated fields of clusters) depend on the matrix partition $(a$ and $b)$, as well as the medium $(u=o$ or $u$ $=i$ ). The number of samples $K_{u}^{1}$, which also depends on the medium, is determined by the excess bandwidth formula. ${ }^{36}$

Radiated fields of clusters in the higher levels of the tree structure are obtained by combining radiated fields of their subclusters. For a cluster $C$ at level $l>1$,

$$
\left\{\begin{array}{l}
\mathbf{S}_{a b, u}^{C, \theta} \\
\mathbf{S}_{a b, u}^{C, \phi}
\end{array}\right\}=\sum_{C^{\prime} \in C} \overline{\boldsymbol{\beta}}_{u}^{C^{\prime} \rightarrow C} \cdot \overline{\boldsymbol{\Gamma}}_{u}^{(l-1) \rightarrow l} \cdot\left\{\begin{array}{l}
\mathbf{S}_{a b, u}^{C^{\prime}, \theta} \\
\mathbf{S}_{a b, u}^{C^{\prime}, \phi}
\end{array}\right\},
$$

where $\overline{\boldsymbol{\beta}}_{u}^{C^{\prime} \rightarrow C}$ is a $K_{u}^{l} \times K_{u}^{l}$ diagonal matrix containing exponential shifts between cluster centers and $\overline{\boldsymbol{\Gamma}}_{u}^{(l-1) \rightarrow l}$ is a $K_{u}^{l}$ $\times K_{u}^{(l-1)}$ sparse interpolation matrix to increase the sampling rate from level $(l-1)$ to $l$.

\subsubsection{Translation and disaggregation}

During the translation and disaggregation stages, incoming fields at cluster centers are calculated from the top of the tree structure to the lowest level. At the highest level, the total incoming field for a cluster is obtained by combining the in- 
coming fields due to translations. At lower levels, however, the incoming field to the center of a cluster involves a contribution from the incoming field to the center of its parent cluster. In general, the total incoming field for a cluster $C$ at level $l$ is obtained as

$$
\begin{aligned}
\left\{\begin{array}{c}
\mathbf{G}_{a b, u}^{C, \theta} \\
\mathbf{G}_{a b, u}^{C, \phi}
\end{array}\right\}= & {\left[\overline{\boldsymbol{\Gamma}}_{u}^{l \rightarrow(l+1)}\right]^{\dagger} \cdot \overline{\boldsymbol{\beta}}_{u}^{P(C) \rightarrow C} \cdot\left\{\begin{array}{l}
\mathbf{G}_{a b, u}^{P(C), \theta} \\
\mathbf{G}_{a b, u}^{P(C), \phi}
\end{array}\right\} } \\
& +\sum_{C^{\prime} \in F(C)} \overline{\mathbf{W}}_{u} \cdot \bar{\alpha}_{u}^{C^{\prime} \rightarrow C} \cdot\left\{\begin{array}{c}
\mathbf{S}_{a b, u}^{C^{\prime}, \theta} \\
\mathbf{S}_{a b, u}^{C^{\prime}, \phi}
\end{array}\right\},
\end{aligned}
$$

where $P(C)$ represents the parent cluster (if it exists), and $F(C)$ represents the clusters that are far from $C$. The first term on the right-hand side of Eq. (36) accounts for a possible contribution from the parent cluster, where $\left[\overline{\boldsymbol{\Gamma}}_{u}^{(l) \rightarrow(l+1)}\right]^{\dagger}$ is a $K_{u}^{l} \times K_{u}^{(l+1)}$ sparse interpolation matrix. The second term represents translations from far-field clusters, where $\overline{\boldsymbol{\alpha}}_{u}^{C^{\prime} \rightarrow C}$ is a diagonal translation matrix, and $\overline{\mathbf{W}}_{u}$ is a diagonal matrix involving integration weights.

Finally, at the lowest level, incoming fields are received by testing functions as

$$
\sum_{n=1}^{N} \overline{\mathbf{Z}}_{a b, u}^{\mathrm{FF}}[m, n] x_{b}[n]=\left(\frac{i k}{4 \pi}\right)^{2}\left\{\mathbf{F}_{a b, u}^{m, \theta} \cdot \mathbf{G}_{a b, u}^{C, \theta}+\mathbf{F}_{a b, u}^{m, \phi} \cdot \mathbf{G}_{a b, u}^{C, \phi}\right\}
$$

for $m \in C$, where $\mathbf{F}_{a b, u}^{m, \theta}$ and $\mathbf{F}_{a b, u}^{m, \phi}$ are arrays of $K_{u}^{1}$ elements containing $\theta$ and $\phi$ components of the receiving pattern of the $m$ th testing function.

\subsection{Preconditioning}

MVMs required by iterative solvers can be performed efficiently by using MLFMA. For an efficient solution, however, the number of iterations should be small, in addition to using fast MVMs. Although JMCFIE provides well-conditioned matrix equations that are easy to solve iteratively, we employ a four-partition block-diagonal preconditioner (4PBDP), which reduces the iteration counts significantly. ${ }^{24}$ Extracting the self-interactions of the lowest level clusters, the matrix equation in Eq. (18) can be preconditioned as

$$
\begin{gathered}
{\left[\begin{array}{ll}
\overline{\mathrm{B}}_{11} & \overline{\mathrm{B}}_{12} \\
\overline{\mathrm{B}}_{21} & \overline{\mathrm{B}}_{22}
\end{array}\right]^{-1} \cdot\left[\begin{array}{ll}
\overline{\mathbf{Z}}_{11} & \overline{\mathbf{Z}}_{12} \\
\overline{\mathbf{Z}}_{21} & \overline{\mathbf{Z}}_{22}
\end{array}\right] \cdot\left[\begin{array}{l}
\mathbf{a}_{J} \\
\mathbf{a}_{M}
\end{array}\right]} \\
=\left[\begin{array}{ll}
\overline{\mathrm{B}}_{11} & \overline{\mathrm{B}}_{12} \\
\overline{\mathrm{B}}_{21} & \overline{\mathrm{B}}_{22}
\end{array}\right]^{-1} \cdot\left[\begin{array}{l}
\mathbf{v}_{1} \\
\mathbf{v}_{2}
\end{array}\right],
\end{gathered}
$$

where $\mathbf{B}_{a b} \approx \overline{\mathbf{Z}}_{a b}$ are block-diagonal matrices. The complexity of $4 \mathrm{PBDP}$ is $\mathcal{O}(N)$.

\section{Solutions and Results}

In this section, we present the solution of scattering problems involving ordinary and deformed RBCs, and we discuss the diagnosis of various diseases using the SCS data. Accuracy of
Table 1 Ordinary and deformed RBCs.

\begin{tabular}{ccccc}
\hline RBC & Volume $\left(\mu \mathrm{m}^{3}\right)$ & $(\mu \mathrm{m})$ & $(\mathrm{deg})$ & $\begin{array}{c}\text { Number } \\
\text { of Unknowns }\end{array}$ \\
\hline Ordinary cell & 80.90 & 7.70 & 14.1 & 213,090 \\
Spherocyte & 80.90 & 5.36 & 9.79 & - \\
Microcyte & 67.42 & 7.24 & 13.2 & 188,472 \\
Macrocyte & 107.9 & 8.48 & 15.5 & 259,086 \\
Sickle cell & 80.90 & 20.9 & 38.1 & 246,828 \\
\hline
\end{tabular}

the numerical results presented here and obtained with JMCFIE, MLFMA, BiCGStab, and 4PBDP is demonstrated in our earlier work. ${ }^{24,37,38}$

\subsection{Modeling of Red Blood Cells}

We consider five different RBCs as illustrated in Fig. 1 and listed in Table 1. An ordinary RBC depicted in Fig. 1(a) is modeled by a rotationally symmetric biconcave surface defined as ${ }^{15}$

$$
r(\theta, \phi)=a \sin ^{q}(\theta)+b,
$$

where $q=5, a=3.3 \mu \mathrm{m}$, and $b=0.55 \mu \mathrm{m}$. With these parameters, the size and the volume of the ordinary RBC are $7.70 \mu \mathrm{m}$ and $80.90 \mu \mathrm{m}^{3}$, respectively. The spherocyte, which is modeled as a dielectric sphere with a 5.36- $\mu \mathrm{m}$ diameter, as depicted in Fig. 1(b), has exactly the same volume as an ordinary RBC. The microcyte and the macrocyte in Figs. 1(c) and 1(d) also have biconcave shapes, as defined in Eq. (39), but their volumes correspond to 5/6 and 4/3 of the volume of an ordinary RBC. Finally, the sickle cell depicted in Fig. 1(e) also has a rotationally symmetric shape as the others do, but it is elongated in one dimension. The size of the sickle cell is $20.9 \mu \mathrm{m}$, whereas its volume is the same as the volume of an ordinary RBC, i.e., $80.90 \mu \mathrm{m}^{3}$.

\subsection{Solutions}

RBCs in Fig. 1 are illuminated by a plane wave propagating in the $-z$ direction with the electric field polarized in the $x$ direction having a unit amplitude. Since the orientation of an RBC can be arbitrary in the flow cytometry, we investigate 13 different cases for each $\mathrm{RBC}$, except for the spherocyte because it has a fully symmetric geometry. As an example, the default orientation $\left(\theta_{o}=0 \mathrm{deg}, \phi_{o}\right.$ $=0 \mathrm{deg}$ ) of an ordinary RBC is depicted in Fig. 2. We perform rotations around the $x$ axis (by an amount of $\theta_{o}$ ) and the $z$ axis (by an amount of $\phi_{o}$ ), respectively. Specifically, we consider $\theta_{o}=\{30 \mathrm{deg}, 60 \mathrm{deg}, 90 \mathrm{deg}\}$ and $\phi_{o}$ $=\{0 \mathrm{deg}, 30 \mathrm{deg}, 60 \mathrm{deg}, 90 \mathrm{deg}\}$, in addition to the default case in Fig. 2.

Relative permittivities of RBCs and the host medium are selected as 1.40 and 1.33 , respectively. Numerical simulations are performed at $474 \mathrm{THz}$, corresponding to the output frequency of a typical helium-neon laser. The size of each RBC 


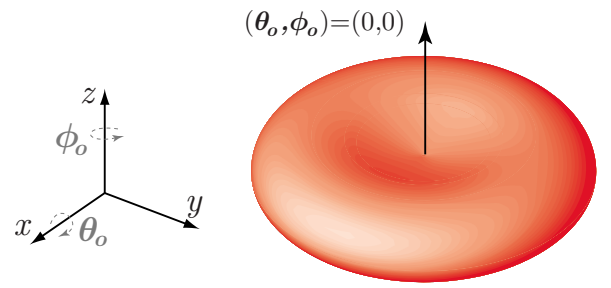

Fig. 2 Default orientation ( $\theta_{0}=0 \mathrm{deg}, \phi_{o}=0 \mathrm{deg}$ ) of an ordinary RBC.

in terms of the wavelength inside the host medium $\left(\lambda_{o}\right)$ are listed in Table 1 . Due to their relatively large sizes, accurate discretizations of RBCs using $\lambda_{o} / 10$ triangles lead to matrix equations involving 180,000 to 260,000 unknowns, as also listed in Table 1. Except for the spherocyte, scattering problems are formulated with JMCFIE and solved iteratively using the BiCGStab algorithm accelerated via MLFMA and 4PBDP, as detailed in Secs. 3 and 4. Scattering from the spherocyte is solved exactly by using a Mie-series algorithm. Solutions with MLFMA require only 15 to 20 iterations, and each solution is performed in 3.0 to $4.5 \mathrm{~h}$ on a $3.6-\mathrm{GHz}$ Intel Xeon processor using 1.5 Gbyte of memory.

\subsection{Results}

Figure 3 presents the solution of scattering problems involving the RBCs depicted in Fig. 1. SCS values in forwardscattering $(\theta=180 \mathrm{deg})$ and back-scattering $(\theta=0 \mathrm{deg})$ directions are plotted in Figs. 3(a) and 3(b), respectively. SCS in a bistatic direction $(\theta, \phi)$ is defined as

$$
\operatorname{SCS}(\theta, \phi)=\lim _{r \rightarrow \infty}\left[4 \pi r^{2} \frac{\left|\mathrm{E}_{o}^{s}(r, \theta, \phi)\right|^{2}}{\left|\mathrm{E}^{\text {inc }}(r, \theta, \phi)\right|^{2}}\right] .
$$

Since SCS depends on the orientation of the target, multiple values are obtained for each cell, except for the spherocyte. We observe that, depending on the cell type, SCS values change significantly. Considering the results for the ordinary $\mathrm{RBC}$, we determine "safe" regions indicated by horizontal lines in Figs. 3(a) and 3(b). For example, an SCS value higher than $44 \mathrm{~dB}_{\mu \mathrm{m}^{2}}$ or lower than $43 \mathrm{~dB} \mu \mathrm{m}^{2}$ in the forwardscattering direction indicates a detection of an abnormal $\mathrm{RBC}$, possibly* a macrocyte (if greater than $44 \mathrm{~dB}^{*} \mathrm{~m}^{2}$ ) or a microcyte (if smaller than $43 \mathrm{~dB}^{2} \mathrm{~m}^{2}$ ). The decibel scale for $\operatorname{SCS}$ is defined as $[\operatorname{SCS}(\theta, \phi)]_{\mathrm{dB}}=10 \log \operatorname{SCS}(\theta, \phi)$. We note that, although less likely, a sickle cell may also present a low SCS value in the forward-scattering direction. As depicted in Fig. 3(b), an SCS value smaller than $-24 \mathrm{~dB} \mu \mathrm{m}^{2}$ in the backscattering direction indicates a detection of a macrocyte, a microcyte, or a sickle cell.

SCS values in forward-scattering and back-scattering directions do not provide complete information for diagnosing diseases. For example, using the data in Figs. 3(a) and 3(b), a spherocyte cannot be distinguished from an ordinary (healthy) cell. The required data can be obtained by considering SCS values in a set of side-scattering directions. We sample SCS

\footnotetext{
* Since SCS has the dimension of area, its unit is square meters. We prefe square micrometers since a single cell has low SCS values. Furthermore, we use decibels square micrometers to improve the visibility of the results on a logarithmic scale.
}

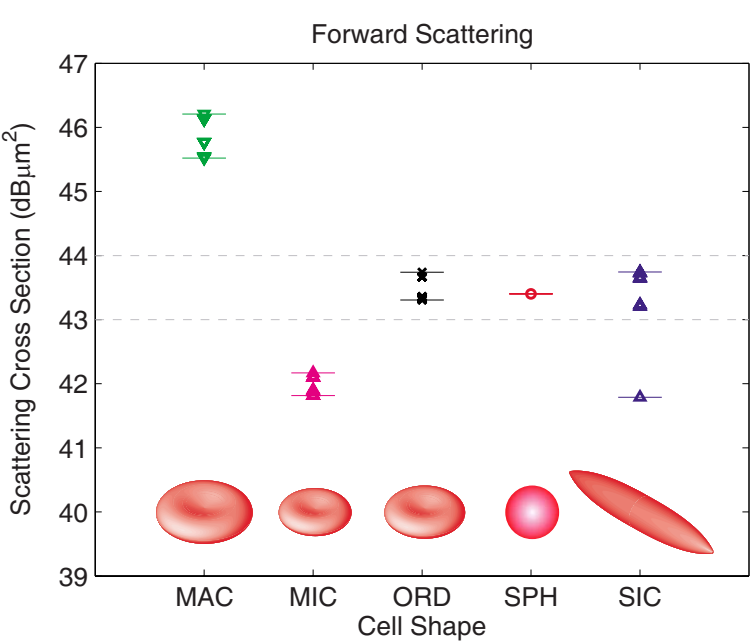

(a)

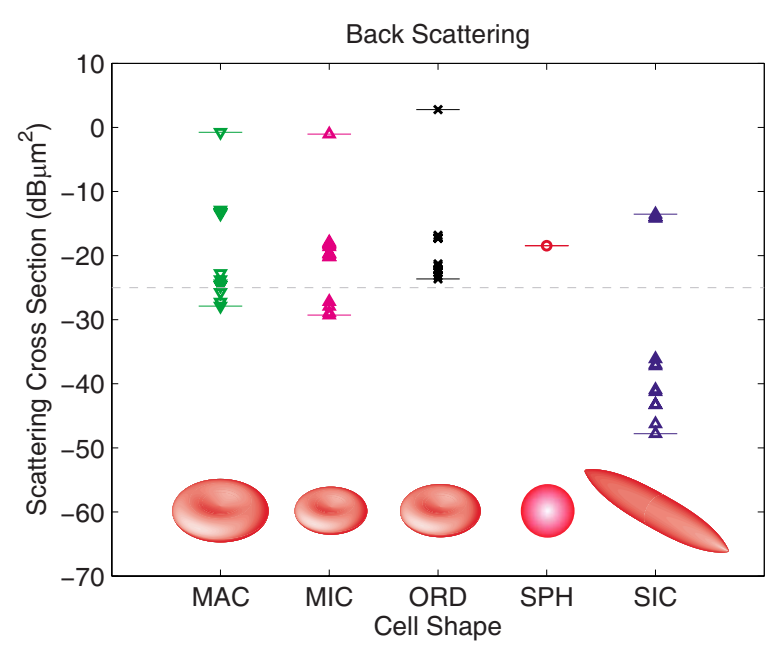

(b)

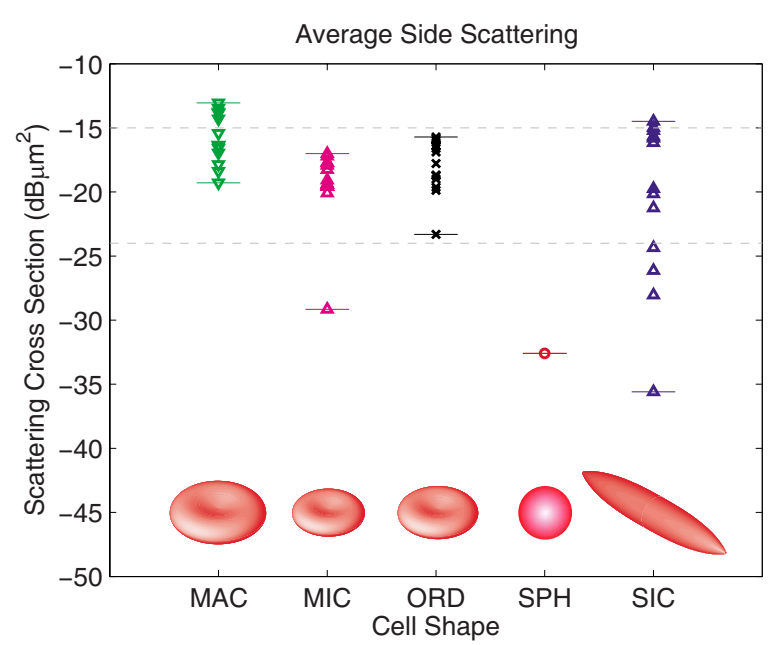

(c)

Fig. 3 Solutions of scattering problems involving an ordinary RBC (ORD) and four different types of deformed RBCs, i.e., a spherocyte $(\mathrm{SPH})$, a macrocyte (MAC), a microcyte (MIC), and a sickle cell (SIC): (a) SCS in the forward-scattering direction, (b) SCS in the backscattering direction, and (c) average SCS in the side-scattering direction are plotted in decibels square micrometer for different orientations of RBCs. 
FORWARD SCATTERING

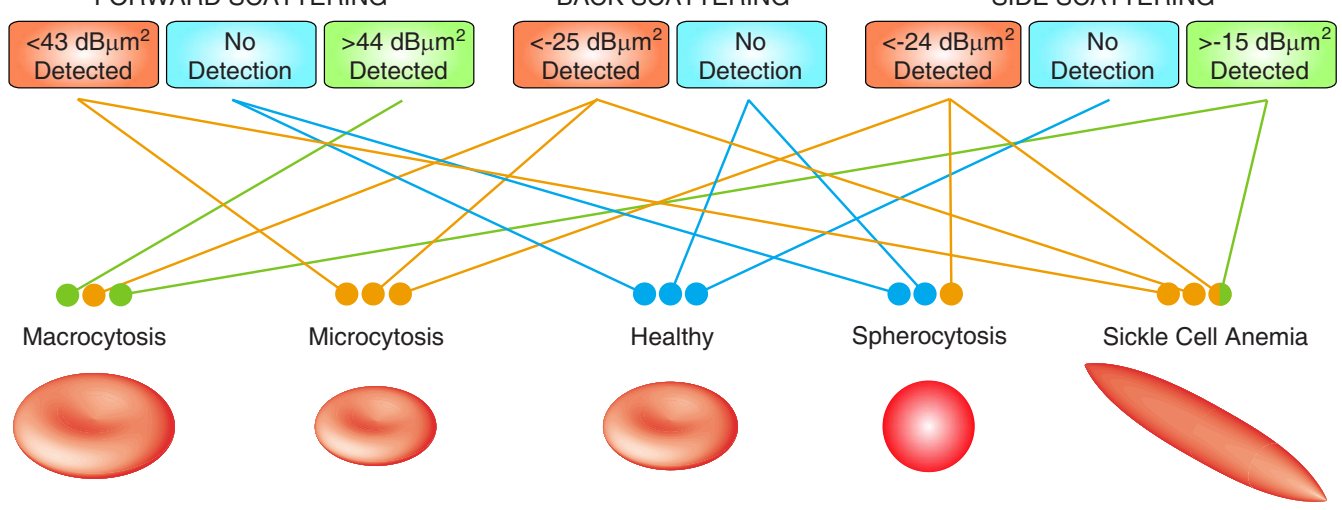

Fig. 4 Decision chart to diagnose various diseases using SCS data.

on the $x-y$ plane as a function of bistatic scattering angle $\phi$ from 0 to $360 \mathrm{deg}$ with 1 -deg intervals. Then, the average $\mathrm{SCS}$ value in the side-scattering direction is computed as

$$
\operatorname{SCS}_{\text {avg }, \text { side }}=\left\{\frac{1}{360} \sum_{n=1}^{360}\left[\left|\operatorname{SCS}\left(\theta=90 \mathrm{deg}, \phi_{n}\right)\right|^{2}\right]\right\}^{1 / 2},
$$

where $\phi_{n}=(n-1) \mathrm{deg}$ for $n=1,2, \ldots, 360$. In the preceding, $\theta=90$ deg refers to the azimuth plane, i.e., the $x$ - $y$ plane, on which all SCS values in the bistatic $\phi_{n}$ directions are averaged. As depicted in Fig. 3(c), an average SCS value higher than $-15 \mathrm{~dB} \mu \mathrm{m}^{2}$ or lower than $-24 \mathrm{~dB} \mu \mathrm{m}^{2}$ in the sidescattering direction indicates a detection of an abnormal RBC, such as a macrocyte, a spherocyte, or a sickle cell.

Finally, using all SCS results in Fig. 3, we determine strict guidelines to detect deformed RBCs in a diagnosis setup. Figure 4 presents a decision chart based on SCS values obtained in forward-scattering, back-scattering, and side-scattering directions. We assume that a sufficient number of RBCs are passed through the setup. The diagnosis of each disease can be described as follows:

1. Macrocytosis: Detection of SCS values higher than $44 \mathrm{~dB} \mu \mathrm{m}^{2}$ in the forward-scattering direction is a major indicator for macrocytosis. SCS values lower than $-25 \mathrm{~dB} \mu \mathrm{m}^{2}$ in the back-scattering direction and average SCS values higher than $-15 \mathrm{~dB} \mu \mathrm{m}^{2}$ in the side-scattering direction increase the reliability of the diagnosis.

2. Microcytosis: Detection of low SCS values in all directions indicates microcytosis. Specifically, detection of SCS values lower than $43 \mathrm{~dB} \mu \mathrm{m}^{2}$ in the forward-scattering direction, SCS values lower than $-25 \mathrm{~dB} \mu \mathrm{m}^{2}$ in the backscattering direction, and average SCS values lower than $-24 \mathrm{~dB} \mu \mathrm{m}^{2}$ in the side-scattering direction indicate microcytosis.

3. Spherocytosis: Spherocytosis can be diagnosed by the detection of average SCS values lower than $-24 \mathrm{~dB} \mu \mathrm{m}^{2}$ in the side-scattering direction, without any abnormal SCS values in the forward-scattering and back-scattering directions.

4. Sickle-Cell Anemia: Similar to microcytosis, sickle-cell anemia can be diagnosed by the detection of low SCS values in all directions. On the other hand, sickle-cell anemia also leads to average SCS values higher than $-15 \mathrm{~dB} \mu \mathrm{m}^{2}$ in the side-scattering direction; this can be used to distinguish sickle-cell anemia from microcytosis.

\section{Conclusion}

We presented a comparative study of scattering from healthy and diseased RBCs with deformed shapes. By using a sophisticated simulation environment based on JMCFIE and MLFMA, scattering problems involving different RBCs with different orientations are solved both accurately and efficiently. By investigating SCS values in forward-scattering, back-scattering, and side-scattering directions, not necessarily limited to conventional cytometer setups, we were able to determine strict guidelines to distinguish deformed RBCs from healthy RBCs and to diagnose related diseases.

\section{Acknowledgments}

This work was supported by the Scientific and Technical Research Council of Turkey (TUBITAK) under Research Grants 105E172 and 107E136, by the Turkish Academy of Sciences in the framework of the Young Scientist Award Program (LG/ TUBA-GEBIP/2002-1-12), and by contracts from ASELSAN and SSM. Özgür Ergür was also supported by a Research Starter Grant provided by the Faculty of Science at the University of Strathclyde.

\section{References}

1. L. O. Reynolds, C. Johnson, and A. Ishimaru, "Diffuse reflectance from a finite blood medium: applications to the modeling of fiber optic catheters," Appl. Opt. 15, 2059-2067 (1976).

2. G. D. Pederson, N. J. McCormick, and L. O. Reynolds, "Transport calculations for light scattering in blood," Biophys. J. 16, 199-207 (1976).

3. R. A. Meyer, "Light scattering from red blood cell ghosts: sensitivity of angular dependent structure to membrane thickness and refractive index," Appl. Opt. 16, 2036-2037 (1977).

4. D. H. Tycko, M. H. Metz, E. A. Epstein, and A. Grinbaum, "Flowcytometric light scattering measurement of red blood cell volume and hemoglobin concentration," Appl. Opt. 24, 1355-1365 (1985).

5. J. M. Steinke and A. P. Shepherd, "Comparison of Mie theory and the light scattering of red blood cells," Appl. Opt. 27, 4027-4033 (1988).

6. G. J. Steekstra, A. G. Hoeksta, E. Nijhof, and R. M. Heethaar, "Light scattering by red blood cells in ektacytometry: Fraunhofer versus anomalous diffraction," Appl. Opt. 32, 2266-2272 (1993).

7. G. J. Streekstra, A. G. Hoekstra, and R. M. Heethaar, "Anomalous diffraction by arbitrarily oriented ellipsoids: applications in ektacytometry," Appl. Opt. 33, 7288-7296 (1994).

8. P. Mazeron and S. Muller, "Dielectric or absorbing particles: EM 
surface fields and scattering," J. Opt. 29, 68-77 (1998).

9. A. G. Borovoi, E. I. Naats, and U. G. Oppel, "Scattering of light by a red blood cell," J. Biomed. Opt. 3, 364-372 (1998).

10. M. Hammer, D. Schweitzer, B. Michel, E. Thamm, and A. Kolb, "Single scattering by red blood cells," Appl. Opt. 37, 7410-7418 (1998).

11. A. N. Shvalov, J. T. Soini, A. V. Chernyshev, P. A. Tarasov, E. Soini, and V. P. Maltsev, "Light-scattering properties of individual erythrocytes," Appl. Opt. 38, 230-235 (1999).

12. A. M. K. Nilsson, P. Alsholm, A. Karlsson, and S. Andersson-Engels, "T-matrix computations of light scattering by red blood cells," Appl. Opt. 37, 2735-2748 (1998).

13. J. He, A. Karlsson, J. Swartling, and S. Andersson-Engels, "Light scattering by multiple red blood cells," J. Opt. Soc. Am. A 21, 1953$1961(2004)$.

14. A. Karlsson, J. He, J. Swartling, and S. Andersson-Engels, "Numerical simulations of light scattering by red blood cells," IEEE Trans. Biomed. Eng. 52, 13-18 (2005).

15. J. Q. Lu, P. Yang, and X. H. Hu, "Simulations of light scattering from a biconcave red blood cell using the finite-difference time-domain method," J. Biomed. Opt. 10, 024022 (2005).

16. N. K. Uzunoglu, D. Yova, and G. S. Stamatakos, "Light scattering by pathological and deformed erythrocytes: an integral equation model," J. Biomed. Opt. 2, 310-318 (1997).

17. G. S. Stamatakos, D. Yova, and N. K. Uzunoglu, "Integral equation model of light scattering by an oriented monodisperse system of triaxial dielectric ellipsoids: application in ektacytometry," Appl. Opt. 36, 6503-6512 (1997)

18. S. V. Tsinopoulos and D. Polyzos, "Scattering of HeNe laser light by an average-sized red blood cell," Appl. Opt. 38, 5499-5510 (1999).

19. S. V. Tsinopoulos, E. J. Sellountos, and D. Polyzos, "Light scattering by aggregated red blood cells," Appl. Opt. 41, 1408-1417 (2002).

20. T. W. Lloyd, J. M. Song, and M. Yang, "Numerical study of surface integral formulations for low-contrast objects," IEEE Antennas Wireless Propag. Lett. 4, 482-485 (2005).

21. J. Song, C.-C. Lu, and W. C. Chew, "Multilevel fast multipole algorithm for electromagnetic scattering by large complex objects," IEEE Trans. Antennas Propag. 45, 1488-1493 (1997).

22. P. Ylä-Oijala and M. Taskinen, "Application of combined field integral equation for electromagnetic scattering by dielectric and composite objects," IEEE Trans. Antennas Propag. 53, 1168-1173 (2005).
23. S. M. Rao, D. R. Wilton, and A. W. Glisson, "Electromagnetic scattering by surfaces of arbitrary shape," IEEE Trans. Antennas Propag. 30, 409-418 (1982).

24. Ö. Ergül and L. Gürel, "Comparison of integral-equation formulations for the fast and accurate solution of scattering problems involving dielectric objects with the multilevel fast multipole algorithm," IEEE Trans. Antennas Propag. 57, 176-187 (2009).

25. A. Tefferi, "Anemia in adults: a contemporary approach to diagnosis," Mayo Clin. Proc. 78, 1274-1280 (2003).

26. L. Pauling, H. A. Itano, S. J. Singer, and I. C. Wells, "Sickle cell anemia, a molecular disease," Science 110, 543-548 (1949).

27. K. H. Walker, W. D. Hall, and J. W. Hurst, Clinical Methods: The History, Physical, and Laboratory Examinations, ButterworthHeinemann, Boston (1990).

28. L. Chrobak, "Microcytic and hypochromic anemias," Vnitr. Lek. 47, 166-174 (2001).

29. F. Aslinia, J. J. Mazza, and S. H. Yale, "Megaloblastic anemia and other causes of macrocytosis," Clin. Med. Res. 4, 236-241 (2006).

30. G. J. Lonergan, D. B. Cline, and S. L. Abbondanzo, "Sickle cell anemia," Radiographics 21, 971-994 (2001).

31. A. Ashley-Koch, Q. Yang, and R. S. Olney, "Sickle hemoglobin (HbS) allele and sickle cell disease: a HuGE review," Am. J. Epidemiol. 151, 839-845 (2000).

32. S. Perrotta, P. G. Gallagher, and N. Mohandas, "Hereditary spherocytosis," Lancet 372, 1411-1426 (2008).

33. American Association for Clinical Chemistry, http:// www.labtestsonline.org.

34. J. A. Stratton, Electromagnetic Theory, McGraw-Hill, New York (1941).

35. H. van der Vorst, "Bi-CGSTAB: a fast and smoothly converging variant of Bi-CG for the solution of nonsymmetric linear systems," SIAM J. Sci. Comput. (USA) 13, 631-644 (1992).

36. W. C. Chew, J.-M. Jin, E. Michielssen, and J. Song, Fast and Efficient Algorithms in Computational Electromagnetics, Artech House, Boston (2001)

37. Ö. Ergül and L. Gürel, "Novel electromagnetic surface integral equations for highly accurate computations of dielectric bodies with arbitrarily low contrasts," J. Comput. Phys. 23, 9898-9912 (2008).

38. Ö. Ergül and L. Gürel, "Efficient solution of the electric-field integral equation by the iterative LSQR algorithm," IEEE Antennas Wireless Propag. Lett. 7, 36-39 (2008). 